Bull. Korean Math. Soc. 48 (2011), No. 1, pp. 95-104

DOI 10.4134/BKMS.2011.48.1.095

\title{
NEW BOUNDS FOR THE OSTROWSKI-LIKE TYPE INEQUALITIES
}

\author{
Vu Nhat Huy AND QuốC-Anh NGô
}

Abstract. We improve some inequalities of Ostrowski-like type and further generalize them.

\section{Introduction}

In 1938, Ostrowski [8] proved the following interesting integral inequality which has received considerable attention from many researchers.

Theorem 1 (See $[8])$. Let $f:[a, b] \rightarrow \mathbb{R}$ be continuous on $[a, b]$ and differentiable on $(a, b)$ whose derivative function $f^{\prime}:(a, b) \rightarrow \mathbb{R}$ is bounded on $(a, b)$, i.e., $\left\|f^{\prime}\right\|_{\infty}=\sup _{t \in(a, b)}\left|f^{\prime}(t)\right|<\infty$. Then

$$
\left|f(x)-\frac{1}{b-a} \int_{a}^{b} f(t) \mathrm{d} t\right| \leqq\left(\frac{1}{4}+\frac{\left(x-\frac{a+b}{2}\right)^{2}}{(b-a)^{2}}\right)(b-a)\left\|f^{\prime}\right\|_{\infty}
$$

for all $x \in[a, b]$.

This inequality gives an upper bound for the approximation of the integral average $\frac{1}{b-a} \int_{a}^{b} f(t) \mathrm{d} t$ by the value $f(x)$ at point $x \in[a, b]$. The first generalization of Ostrowski inequality was given by G. V. Milovanović and J. E. Pečarić in [7]. However, note that estimate (1) can be applied only if $f^{\prime}$ is bounded. In the first part of this paper, we will improve (1) by assuming $f^{\prime} \in L^{p}(a, b)$ for some $1 \leqq p<\infty$. More precisely, we obtain the following theorem.

Theorem 2. Assume that $1 \leqq p$. Let $I \subset \mathbb{R}$ be an open interval such that $[a, b] \subset I$ and let $f: I \rightarrow \mathbb{R}$ be a differentiable function such that $f^{\prime} \in L^{p}(a, b)$. Then we have

$$
\left|f(x)-\frac{1}{b-a} \int_{a}^{b} f(t) \mathrm{d} t\right| \leqq A(x, q)\left\|f^{\prime}\right\|_{p}
$$

Received May 13, 2009.

2010 Mathematics Subject Classification. 26D10, 41A55, 65D30.

Key words and phrases. inequality, error, integral, Taylor, Ostrowski. 
for all $x \in[a, b]$ where

$$
A(x, q)=\left(\frac{1}{b-a}\left(\frac{1}{q+1}\left(\frac{b-a}{2}\right)^{q+1}\right)^{\frac{1}{q}}+\left|x-\frac{a+b}{2}\right|^{\frac{1}{q}}\right)
$$

and $\frac{1}{p}+\frac{1}{q}=1$.

Remark 1. $\lim _{q \rightarrow+\infty} A(x, q)=\frac{3}{2}$ for each $x \in[a, b]$.

Example 1. Let us consider the integral

$$
\int_{0}^{1} \sqrt[3]{\sin \left(t^{2}\right)} \mathrm{d} t
$$

Then we have

$$
f(t)=\sqrt[3]{\sin \left(t^{2}\right)} \quad \text { and } \quad f^{\prime}(t)=\frac{2 t \cos \left(t^{2}\right)}{3 \sqrt[3]{\sin ^{2}\left(t^{2}\right)}}
$$

such that $f^{\prime}(t) \rightarrow \infty$ as $t \rightarrow 0$. On the other hand, we have

$$
\int_{0}^{1}\left|f^{\prime}(t)\right|^{2} \mathrm{~d} t \leqq \frac{4}{9} \max _{0 \leqq t \leqq 1}\left|\frac{t^{2} \cos \left(t^{2}\right)}{\sin \left(t^{2}\right)}\right| \int_{0}^{1} \frac{\mathrm{d} t}{\sqrt[3]{\sin \left(t^{2}\right)}} \leqq \frac{16}{9},
$$

i.e., $\left\|f^{\prime}\right\|_{L^{2}} \leqq \frac{4}{3}$. It follows that

$$
\left|\sqrt[3]{\sin \left(x^{2}\right)}-\int_{0}^{1} \sqrt[3]{\sin \left(t^{2}\right)} \mathrm{d} t\right| \leqq \frac{4}{3}\left(\frac{1}{\sqrt{24}}+\sqrt{x-\frac{1}{2}}\right)
$$

for all $x \in[0,1]$.

In recent years, a number of authors have written about generalizations of Ostrowski inequality. For example, this topic is considered in $[1,3,4,6,11,5]$. In this way, some new types of inequalities are formed, such as inequalities of Ostrowski-Griiss type, inequalities of Ostrowski-Chebyshev type, etc. The first inequality of Ostrowski-Grüss type was given by Dragomir and Wang in [4]. It was generalized and improved by Matić, Pečarić, and Ujević in [6]. Cheng gave a sharp version of the mentioned inequality in [3]. Recently in [11], Ujević proved the following result which gives much better results than estimations based on [3].

Theorem 3 (See $[11$, Theorem 4]). Let $f: I \rightarrow \mathbb{R}$, where $I \subset \mathbb{R}$ is an interval, be a twice continuously differentiable mapping in the interior $\stackrel{\circ}{I}$ of $I$ with $f^{\prime \prime} \in$ $L^{2}(a, b)$ and let $a, b \in \stackrel{\circ}{I}, a<b$. Then we have

$$
\left|f(x)-\frac{1}{b-a} \int_{a}^{b} f(t) \mathrm{d} t-\left(x-\frac{a+b}{2}\right) \frac{f(b)-f(a)}{b-a}\right| \leqq \frac{(b-a)^{\frac{3}{2}}}{2 \pi \sqrt{3}}\left\|f^{\prime \prime}\right\|_{2}
$$

for all $x \in[a, b]$. 
If we assume $f$ is such that $f^{\prime \prime}$ is of class $L^{p}$ for some $1 \leqq p<\infty$, then we obtain:

Theorem 4. Let $f: I \rightarrow \mathbb{R}$, where $I \subset \mathbb{R}$ is an interval, be a twice continuously differentiable mapping in the interior $\stackrel{\circ}{I}$ of $I$ with $f^{\prime \prime} \in L^{p}(a, b), 1 \leqq p<\infty$, we have

$$
\left|f(x)-\frac{1}{b-a} \int_{a}^{b} f(t) \mathrm{d} t-\left(x-\frac{a+b}{2}\right) \frac{f(b)-f(a)}{b-a}\right| \leqq B(q)\left\|f^{\prime \prime}\right\|_{p}
$$

for all $x \in[a, b]$ where

$$
B(q)=\left[\frac{3}{2}\left(\frac{(b-a)^{q+1}}{q+1}\right)^{\frac{1}{q}}+\frac{1}{2(b-a)}\left(\frac{(b-a)^{2 q+1}}{2 q+1}\right)^{\frac{1}{q}}\right]
$$

and $\frac{1}{p}+\frac{1}{q}=1$.

Remark 2. $\lim _{q \rightarrow+\infty} B(q)=2(b-a)$.

\section{Proofs}

Before proving our main theorem, we need an essential lemma below. It is well-known in the literature as Taylor's formula or Taylor's theorem with the integral remainder.

Lemma 5 (See $[2])$. Let $f:[a, b] \rightarrow \mathbb{R}$ and let $r$ be a positive integer. If $f$ is such that $f^{(r-1)}$ is absolutely continuous on $[a, b], x_{0} \in(a, b)$, then for all $x \in(a, b)$ we have

$$
f(x)=T_{r-1}\left(f, x_{0}, x\right)+R_{r-1}\left(f, x_{0}, x\right),
$$

where $T_{r-1}\left(f, x_{0}, \cdot\right)$ is a Taylor's polynomial of degree $r-1$, that is,

$$
T_{r-1}\left(f, x_{0}, x\right)=\sum_{k=0}^{r-1} \frac{f^{(k)}\left(x_{0}\right)\left(x-x_{0}\right)^{k}}{k !}
$$

and the remainder can be given by

$$
R_{r-1}\left(f, x_{0}, x\right)=\int_{x_{0}}^{x} \frac{(x-t)^{r-1} f^{(r)}(t)}{(r-1) !} \mathrm{d} t .
$$

By a simple calculation, the remainder in (5) can be rewritten as

$$
R_{r-1}\left(f, x_{0}, x\right)=\int_{0}^{x-x_{0}} \frac{\left(x-x_{0}-t\right)^{r-1} f^{(r)}\left(x_{0}+t\right)}{(r-1) !} \mathrm{d} t
$$

which helps us to deduce a similar representation of $f$ as following

$$
f(x+u)=\sum_{k=0}^{r-1} \frac{u^{k}}{k !} f^{(k)}(x)+\int_{0}^{u} \frac{(u-t)^{r-1}}{(r-1) !} f^{(r)}(x+t) \mathrm{d} t .
$$


Proof of Theorem 2. Denote

$$
F(x)=\int_{a}^{x} f(t) \mathrm{d} t
$$

By Fundamental Theorem of Calculus

$$
I(f)=F(b)-F(a) .
$$

Applying Lemma 5 gives

$$
F(b)=F\left(\frac{a+b}{2}\right)+\frac{b-a}{2} F^{\prime}\left(\frac{a+b}{2}\right)+\int_{\frac{a+b}{2}}^{b}(b-t) F^{\prime \prime}(t) \mathrm{d} t
$$

which implies that

$$
F(b)-F\left(\frac{a+b}{2}\right)=\frac{b-a}{2} f\left(\frac{a+b}{2}\right)+\int_{\frac{a+b}{2}}^{b}(b-t) f^{\prime}(t) \mathrm{d} t .
$$

We see that

$$
F(a)=F\left(\frac{a+b}{2}\right)+\frac{a-b}{2} F^{\prime}\left(\frac{a+b}{2}\right)+\int_{\frac{a+b}{2}}^{a}(a-t) F^{\prime \prime}(t) \mathrm{d} t
$$

which yields

$$
F(a)-F\left(\frac{a+b}{2}\right)=\frac{a-b}{2} f\left(\frac{a+b}{2}\right)+\int_{a}^{\frac{a+b}{2}}(t-a) f^{\prime}(t) \mathrm{d} t .
$$

Therefore,

$F(b)-F(a)=(b-a) f\left(\frac{a+b}{2}\right)+\int_{\frac{a+b}{2}}^{b}(b-t) f^{\prime}(t) \mathrm{d} t-\int_{a}^{\frac{a+b}{2}}(t-a) f^{\prime}(t) \mathrm{d} t$.

By changing $t=a+b-x$, we get

$$
\int_{\frac{a+b}{2}}^{b}(b-t) f^{\prime}(t) \mathrm{d} t=\int_{a}^{\frac{a+b}{2}}(t-a) f^{\prime}(a+b-t) \mathrm{d} t
$$

which helps us to deduce that

$$
\int_{a}^{b} f(t) \mathrm{d} t=(b-a) f\left(\frac{a+b}{2}\right)+\int_{a}^{\frac{a+b}{2}}(t-a)\left(f^{\prime}(a+b-t)-f^{\prime}(t)\right) \mathrm{d} t .
$$

On the other hand,

$$
f(x)-f\left(\frac{a+b}{2}\right)=\int_{\frac{a+b}{2}}^{x} f^{\prime}(t) \mathrm{d} t
$$

Then

$$
\begin{aligned}
& f(x)-\frac{1}{b-a} \int_{a}^{b} f(t) \mathrm{d} t \\
= & \int_{\frac{a+b}{2}}^{x} f^{\prime}(t) \mathrm{d} t-\frac{1}{b-a} \int_{a}^{\frac{a+b}{2}}(t-a)\left(f^{\prime}(a+b-t)-f^{\prime}(t)\right) \mathrm{d} t .
\end{aligned}
$$


Next we consider the case $1<p<\infty$. We first have the following estimates

$$
\begin{aligned}
& \left|\int_{a}^{\frac{a+b}{2}}(t-a)\left(f^{\prime}(a+b-t)-f^{\prime}(t)\right) \mathrm{d} t\right| \\
\leqq & \left|\int_{a}^{\frac{a+b}{2}}(t-a) f^{\prime}(a+b-t) \mathrm{d} t\right|+\left|\int_{a}^{\frac{a+b}{2}}(t-a) f^{\prime}(t) \mathrm{d} t\right| \\
\leqq & \left(\int_{a}^{\frac{a+b}{2}}\left|f^{\prime}(a+b-t)\right|^{p} \mathrm{~d} t\right)^{\frac{1}{p}}\left(\int_{a}^{\frac{a+b}{2}}|t-a|^{q} \mathrm{~d} t\right)^{\frac{1}{q}} \\
& +\left(\int_{a}^{\frac{a+b}{2}}\left|f^{\prime}(t)\right|^{p} \mathrm{~d} t\right)^{\frac{1}{p}}\left(\int_{a}^{\frac{a+b}{2}}|t-a|^{q} \mathrm{~d} t\right)^{\frac{1}{q}} \\
= & \left(\frac{1}{q+1}\left(\frac{b-a}{2}\right)^{q+1}\right)^{\frac{1}{q}}\left\|f^{\prime}\right\|_{p} .
\end{aligned}
$$

Clearly,

$$
\begin{aligned}
\left|\int_{\frac{a+b}{2}}^{x} f^{\prime}(t) \mathrm{d} t\right| & \leqq\left.\left.\left|\int_{\frac{a+b}{2}}^{x}\right| f^{\prime}(t)\right|^{p} \mathrm{~d} t|| \int_{\frac{a+b}{2}}^{\frac{1}{p}} 1^{q} \mathrm{~d} t\right|^{\frac{1}{q}} \\
& \leqq\left.\left.\left|\int_{a}^{b}\right| f^{\prime}(t)\right|^{p} \mathrm{~d} t\right|^{\frac{1}{p}}\left|\int_{\frac{a+b}{2}}^{x} 1^{q} \mathrm{~d} t\right|^{\frac{1}{q}} \\
& =\left\|f^{\prime}\right\|_{p}\left|x-\frac{a+b}{2}\right|^{\frac{1}{q}}
\end{aligned}
$$

Hence,

$$
\begin{aligned}
& \left|f(x)-\frac{1}{b-a} \int_{a}^{b} f(t) \mathrm{d} t\right| \\
\leqq & \left(\frac{1}{b-a}\left(\frac{1}{q+1}\left(\frac{b-a}{2}\right)^{q+1}\right)^{\frac{1}{q}}+\left|x-\frac{a+b}{2}\right|^{\frac{1}{q}}\right)\left\|f^{\prime}\right\|_{p} .
\end{aligned}
$$

If $p=1$, then

$$
\begin{aligned}
& \left|\int_{a}^{\frac{a+b}{2}}(t-a)\left(f^{\prime}(a+b-t)-f^{\prime}(t)\right) \mathrm{d} t\right| \\
\leqq & \frac{b-a}{2} \int_{a}^{\frac{a+b}{2}}\left(\left|f^{\prime}(a+b-t)\right|+\left|f^{\prime}(t)\right|\right) \mathrm{d} t \\
= & \frac{b-a}{2}\left\|f^{\prime}\right\|_{1}
\end{aligned}
$$


and

which helps us to claim that

$$
\left|\int_{\frac{a+b}{2}}^{x} f^{\prime}(t) \mathrm{d} t\right| \leqq\left\|f^{\prime}\right\|_{1}
$$

$$
\left|f(x)-\frac{1}{b-a} \int_{a}^{b} f(t) \mathrm{d} t\right| \leqq \frac{3}{2}\left\|f^{\prime}\right\|_{1} .
$$

Corollary 1. If we put $x=\frac{a+b}{2}$, then under the assumptions of Theorem 1 and $1 \leqq p<\infty$, we have

$$
\left|f\left(\frac{a+b}{2}\right)-\frac{1}{b-a} \int_{a}^{b} f(t) \mathrm{d} t\right| \leqq \frac{1}{b-a}\left(\frac{1}{q+1}\left(\frac{b-a}{2}\right)^{q+1}\right)^{\frac{1}{q}}\left\|f^{\prime}\right\|_{p} .
$$

Note that

$$
\frac{1}{b-a}\left(\frac{1}{q+1}\left(\frac{b-a}{2}\right)^{q+1}\right)^{\frac{1}{q}}=\frac{1}{2}\left(\frac{1}{q+1}\right)^{\frac{1}{q}}\left(\frac{b-a}{2}\right)^{\frac{1}{q}} .
$$

Proof of Theorem 4. Clearly, by Lemma 5 one has

$$
\begin{aligned}
\frac{1}{b-a} \int_{a}^{b} f(t) \mathrm{d} t & =\frac{1}{b-a}(F(b)-F(a)) \\
& =\frac{1}{b-a}\left((b-a) F^{\prime}(a)+\frac{(b-a)^{2}}{2} F^{\prime \prime}(a)+\int_{a}^{b} \frac{(b-t)^{2}}{2} F^{\prime \prime \prime}(t) \mathrm{d} t\right) \\
& =f(a)+\frac{b-a}{2} f^{\prime}(a)+\frac{1}{b-a} \int_{a}^{b} \frac{(b-x)^{2}}{2} f^{\prime \prime}(t) \mathrm{d} t .
\end{aligned}
$$

Similarly,

$$
f(x)=f(a)+(x-a) f^{\prime}(a)+\int_{a}^{b}(b-t) f^{\prime \prime}(t) \mathrm{d} t
$$

and

$$
\begin{aligned}
\frac{f(b)-f(a)}{b-a} & =\frac{1}{b-a}\left((b-a) f^{\prime}(a)+\int_{a}^{b}(b-t) f^{\prime \prime}(t) \mathrm{d} t\right) \\
& =f^{\prime}(a)+\frac{1}{b-a} \int_{a}^{b}(b-t) f^{\prime \prime}(t) \mathrm{d} t
\end{aligned}
$$

Therefore,

$$
\begin{aligned}
& \left|f(x)-\frac{1}{b-a} \int_{a}^{b} f(t) \mathrm{d} t-\left(x-\frac{a+b}{2}\right) \frac{f(b)-f(a)}{b-a}\right| \\
= & \left|\int_{a}^{b}(b-x) f^{\prime \prime}(x) \mathrm{d} t-\frac{1}{b-a} \int_{a}^{b} \frac{(b-t)^{2}}{2} f^{\prime \prime}(t) \mathrm{d} t-\frac{x-\frac{a+b}{2}}{b-a} \int_{a}^{b}(b-t) f^{\prime \prime}(t) \mathrm{d} t\right| .
\end{aligned}
$$


If $1<p<\infty$, then by the Hölder inequality, one has

$$
\left|\int_{a}^{b}(b-t) f^{\prime \prime}(t) \mathrm{d} t\right| \leqq\left\|f^{\prime \prime}\right\|_{p}\left(\int_{a}^{b}(b-t)^{q} \mathrm{~d} t\right)^{\frac{1}{q}}=\left(\frac{(b-a)^{q+1}}{q+1}\right)^{\frac{1}{q}}\left\|f^{\prime \prime}\right\|_{p}
$$

and

$$
\begin{aligned}
\frac{1}{b-a}\left|\int_{a}^{b} \frac{(b-t)^{2}}{2} f^{\prime \prime}(t) \mathrm{d} t\right| & \leqq \frac{1}{2(b-a)}\left\|f^{\prime \prime}\right\|_{p}\left(\int_{a}^{b}(b-t)^{2 q} \mathrm{~d} t\right)^{\frac{1}{q}} \\
& =\frac{1}{2(b-a)}\left(\frac{(b-a)^{2 q+1}}{2 q+1}\right)^{\frac{1}{q}}\left\|f^{\prime \prime}\right\|_{p}
\end{aligned}
$$

and

$$
\begin{aligned}
\left|\frac{x-\frac{a+b}{2}}{b-a} \int_{a}^{b}(b-t) f^{\prime \prime}(t) \mathrm{d} t\right| & \leqq \frac{1}{2}\left|\int_{a}^{b}(b-t) f^{\prime \prime}(t) \mathrm{d} t\right| \\
& \leqq \frac{1}{2}\left(\frac{(b-a)^{q+1}}{q+1}\right)^{\frac{1}{q}}\left\|f^{\prime \prime}\right\|_{p} .
\end{aligned}
$$

Thus,

$$
\begin{aligned}
& \left|f(x)-\frac{1}{b-a} \int_{a}^{b} f(t) \mathrm{d} t-\left(x-\frac{a+b}{2}\right) \frac{f(b)-f(a)}{b-a}\right| \\
\leqq & {\left[\frac{3}{2}\left(\frac{(b-a)^{q+1}}{q+1}\right)^{\frac{1}{q}}+\frac{1}{2(b-a)}\left(\frac{(b-a)^{2 q+1}}{2 q+1}\right)^{\frac{1}{q}}\right]\left\|f^{\prime \prime}\right\|_{p} . }
\end{aligned}
$$

If $1=p$, then again by the Hölder inequality, one has

$$
\left|\int_{a}^{b}(b-t) f^{\prime \prime}(t) \mathrm{d} t\right| \leqq(b-a) \int_{a}^{b}\left|f^{\prime \prime}(t)\right| \mathrm{d} t=(b-a)\left\|f^{\prime \prime}\right\|_{1},
$$

and

$$
\frac{1}{b-a}\left|\int_{a}^{b} \frac{(b-t)^{2}}{2} f^{\prime \prime}(t) \mathrm{d} t\right| \leqq \frac{1}{b-a} \frac{(b-a)^{2}}{2} \int_{a}^{b}\left|f^{\prime \prime}(t)\right| \mathrm{d} t=\frac{b-a}{2}\left\|f^{\prime \prime}\right\|_{1},
$$

and

$$
\left|\frac{x-\frac{a+b}{2}}{b-a} \int_{a}^{b}(b-t) f^{\prime \prime}(t) \mathrm{d} t\right| \leqq \frac{1}{2}\left|\int_{a}^{b}(b-t) f^{\prime \prime}(t) \mathrm{d} t\right| \leqq \frac{1}{2}(b-a)\left\|f^{\prime \prime}\right\|_{1} .
$$

Hence,

$$
\left|f(x)-\frac{1}{b-a} \int_{a}^{b} f(t) \mathrm{d} t-\left(x-\frac{a+b}{2}\right) \frac{f(b)-f(a)}{b-a}\right| \leqq 2(b-a)\left\|f^{\prime \prime}\right\|_{1} .
$$


Therefore,

$$
\begin{aligned}
& \left|f(x)-\frac{1}{b-a} \int_{a}^{b} f(t) \mathrm{d} t-\left(x-\frac{a+b}{2}\right) \frac{f(b)-f(a)}{b-a}\right| \\
\leqq & {\left[\frac{3}{2}\left(\frac{(b-a)^{q+1}}{q+1}\right)^{\frac{1}{q}}+\frac{1}{2(b-a)}\left(\frac{(b-a)^{2 q+1}}{2 q+1}\right)^{\frac{1}{q}}\right]\left\|f^{\prime \prime}\right\|_{p} . }
\end{aligned}
$$

Corollary 2. If we put $x=\frac{a+b}{2}$, then under the assumptions of Theorem 3 and $1 \leqq p<\infty$, we have

$$
\begin{aligned}
& \left|f\left(\frac{a+b}{2}\right)-\frac{1}{b-a} \int_{a}^{b} f(t) \mathrm{d} t\right| \\
\leqq & {\left[\frac{3}{2}\left(\frac{(b-a)^{q+1}}{q+1}\right)^{\frac{1}{q}}+\frac{1}{2(b-a)}\left(\frac{(b-a)^{2 q+1}}{2 q+1}\right)^{\frac{1}{q}}\right]\left\|f^{\prime \prime}\right\|_{p} . }
\end{aligned}
$$

\section{Applications in numerical integral}

Let $\Gamma=\left\{x_{0}=a<x_{1}<\cdots<x_{n}=b\right\}$ be a given subdivision of the interval $[a, b]$ such that $h=x_{i+1}-x_{i}=\frac{b-a}{n}$. Then we obtain the following theorem by using Corollary 1.

Theorem 6. Under the assumptions of Theorem 2 and $1 \leqq p<\infty$, we have

$$
\left|\frac{1}{n} \sum_{i=1}^{n} f\left(\frac{x_{i-1}+x_{i}}{2}\right)-\frac{1}{b-a} \int_{a}^{b} f(t) \mathrm{d} t\right| \leqq \frac{1}{2 n}\left(\frac{1}{q+1}\right)^{\frac{1}{q}}\left(\frac{b-a}{2}\right)^{\frac{1}{q}}\left\|f^{\prime}\right\|_{p} .
$$

Proof. We have

$$
\left|f\left(\frac{x_{i-1}+x_{i}}{2}\right)-\frac{n}{b-a} \int_{x_{i-1}}^{x_{i}} f(t) \mathrm{d} t\right| \leqq \frac{1}{2}\left(\frac{1}{q+1}\right)^{\frac{1}{q}}\left(\frac{b-a}{2 n}\right)^{\frac{1}{q}}\left\|f^{\prime}\right\|_{p,\left[x_{i-1}, x_{i}\right]}
$$

where

$$
\left\|f^{\prime}\right\|_{p,\left[x_{i-1}, x_{i}\right]}=\left(\int_{x_{i-1}}^{x_{i}}\left|f^{\prime}(t)\right|^{p} \mathrm{~d} t\right)^{\frac{1}{p}}
$$

Then,

$$
\begin{aligned}
& \left|\frac{1}{n} \sum_{i=1}^{n} f\left(\frac{x_{i-1}+x_{i}}{2}\right)-\frac{1}{b-a} \int_{a}^{b} f(t) \mathrm{d} t\right| \\
\leqq & \frac{1}{2 n}\left(\frac{1}{q+1}\right)^{\frac{1}{q}}\left(\frac{b-a}{2 n}\right)^{\frac{1}{q}} \sum_{i=1}^{n}\left\|f^{\prime}\right\|_{p,\left[x_{i-1}, x_{i}\right]} .
\end{aligned}
$$

Put

$$
\alpha_{i}=\int_{x_{i-1}}^{x_{i}}\left|f^{\prime}(t)\right|^{p} \mathrm{~d} t
$$


Then

$$
\sum_{i=1}^{n}\left\|f^{\prime}\right\|_{p,\left[x_{i-1}, x_{i}\right]}=\sum_{i=1}^{n} \alpha_{i}^{\frac{1}{p}} \leqq n^{1-\frac{1}{p}}\left(\sum_{i=1}^{n} \alpha_{i}\right)^{\frac{1}{p}}=n^{1-\frac{1}{p}}\left\|f^{\prime}\right\|_{p} .
$$

Therefore,

$$
\begin{aligned}
& \left|\frac{1}{n} \sum_{i=1}^{n} f\left(\frac{x_{i-1}+x_{i}}{2}\right)-\frac{1}{b-a} \int_{a}^{b} f(t) \mathrm{d} t\right| \\
\leqq & \frac{1}{2 n}\left(\frac{1}{q+1}\right)^{\frac{1}{q}}\left(\frac{b-a}{2 n}\right)^{\frac{1}{q}} n^{1-\frac{1}{p}}\left\|f^{\prime}\right\|_{p} \\
= & \frac{1}{2 n}\left(\frac{1}{q+1}\right)^{\frac{1}{q}}\left(\frac{b-a}{2}\right)^{\frac{1}{q}}\left\|f^{\prime}\right\|_{p} .
\end{aligned}
$$

If we use Corollary 2, we then obtain the following theorem whose proof will be omitted.

Theorem 7. Under the assumptions of Theorem 4 and $1 \leqq p<\infty$, we have

$$
\begin{aligned}
& \left|\frac{1}{n} \sum_{i=1}^{n} f\left(\frac{x_{i-1}+x_{i}}{2}\right)-\frac{1}{b-a} \int_{a}^{b} f(t) \mathrm{d} t\right| \\
\leqq & \frac{1}{n^{2}}\left[\frac{3}{2}\left(\frac{(b-a)^{q+1}}{q+1}\right)^{\frac{1}{q}}+\frac{1}{2(b-a)}\left(\frac{(b-a)^{2 q+1}}{2 q+1}\right)^{\frac{1}{q}}\right]\left\|f^{\prime \prime}\right\|_{p} .
\end{aligned}
$$

\section{References}

[1] G. A. Anastassiou, Ostrowski type inequalities, Proc. Amer. Math. Soc. 123 (1995), no. $12,3775-3781$.

[2] G. A. Anastassiou and S. S. Dragomir, On some estimates of the remainder in Taylor's formula, J. Math. Anal. Appl. 263 (2001), no. 1, 246-263.

[3] X. L. Cheng, Improvement of some Ostrowski-Grüss type inequalities, Comput. Math. Appl. 42 (2001), no. 1-2, 109-114.

[4] S. S. Dragomir and S. Wang, An inequality of Ostrowski-Gruss type and its applications to the estimation of error bounds for some special means and for some numerical quadrature rules, Comput. Math. Appl. 33 (1997), no. 11, 15-20.

[5] V. N. Huy and Q. A. Ngô, New inequalities of Ostrowski-like type involving $n$ knots and the $L^{p}$-norm of the m-th derivative, Appl. Math. Lett. 22 (2009), no. 9, 1345-1350.

[6] M. Matić, J. E. Pečarić, and N. Ujević, Improvement and further generalization of inequalities of Ostrowski-Grüss type, Comput. Math. Appl. 39 (2000), no. 3-4, 161-175.

[7] G. V. Milovanović and J. E. Pečarić, On generalization of the inequality of A. Ostrowski and some related applications, Univ. Beograd. Publ. Elektrotehn. Fak. Ser. Mat. Fiz. No. 544-576 (1976), 155-158.

[8] A. M. Ostrowski, Über die absolutabweichung einer differentiebaren funktion von ihrem integralmitelwert, Comment. Math. Helv. 10 (1938), 226-227.

[9] N. Ujević, Error inequalities for a quadrature formula of open type, Rev. Colombiana Mat. 37 (2003), no. 2, 93-105. 
[10] Error inequalities for a quadrature formula and applications, Comput. Math. Appl. 48 (2004), no. 10-11, 1531-1540.

[11] _ New bounds for the first inequality of Ostrowski-Grüss type and applications, Comput. Math. Appl. 46 (2003), no. 2-3, 421-427.

Vu Nhat HuY

Department of Mathematics

College of SCience

Viêt Nam National University

HÀ NôI, VIÊT NAM

E-mail address: nhat_huy85@yahoo.com

QuốC-ANH NGÔ

College of Science

Viêt Nam National University

HÀ NôI, VIÊT NAM

AND

Department of Mathematics

National University of Singapore

Block S17 (SOC1), 10 Lower Kent Ridge RoAd

119076, Singapore

E-mail address: bookworm_vn@yahoo.com 